
\title{
28 Research Square \\ Survival and Prognostic Factors Analyses in Malignant Giant Cell Tumor of Bone
}

\section{Jin Zhang}

Tianjin Tumor Hospital

\section{Xin Wang}

First Affiliated Hospital, Army Medical University

\section{Feng Lin}

Tianjin Tumor Hospital

\section{Guijun Xu}

Tianjin Tumor Hospital

\section{Haixiao Wu}

Tianjin Tumor Hospital

\section{Jincai Duan}

Tianjin Tumor Hospital

\section{Min Mao}

First Affiliated Hospital, Army Medical University

Chao Zhang ( $\nabla$ drzhangchao@tmu.edu.cn )

Tianjin Tumor Hospital https://orcid.org/0000-0001-7096-8488

\section{Research article}

Keywords: giant cell tumour of bone, malignant, survival, prognosis, SEER

Posted Date: August 7th, 2020

DOI: https://doi.org/10.21203/rs.3.rs-54236/v1

License: (c) (1) This work is licensed under a Creative Commons Attribution 4.0 International License. Read Full License 


\section{Abstract}

Background: The characteristics and survival of patients with malignant giant cell tumour of bone (GCTB) have not been investigated thoroughly due to the rarity of the disease. We evaluated these factors in a large cohort in the National Cancer Institute's Surveillance, Epidemiology, and End Results (SEER) database.

Methods: Data from patients who were diagnosed with malignant GCTB from 1975 to 2016 were extracted from the SEER database. The overall survival was calculated by Kaplan-Meier analysis, and intergroup differences were tested by the log-rank test. Univariate and multivariate Cox proportional hazard regression analyses were conducted to identify the independent survival factors.

Results: A total of 325 patients with malignant GCTB were included. The overall 1-, 5-, and 10-year survival rates were $94.3 \%$ (95\% Cl: 91.7-96.8), 82.3\% (95\% Cl: 77.9-86.6), and 80.1\% (95\% Cl: 75.4-84.7), respectively. A potential non-linear $\mathrm{J}$-shaped dose-response relationship between the age or diagnosis year and survival were found. Multivariate Cox regression showed poor survival in patients with age from 35 to 60 years (HR=9.99, 95\% Cl: 1.34-74.80, $P=0.025)$, age older than 60 years (HR=62.03, 95\% Cl: 7.94484.38, $P<0.001$ ), with stage T2 disease ( $\mathrm{HR}=4.85,95 \% \mathrm{Cl}$ : 1.52-15.47, $P=0.008)$, with stage T3 disease ( $\mathrm{HR}=6.09,95 \% \mathrm{Cl}: 1.03-36.23, P=0.047)$, and with distant tumours (HR=2.76, 95\% $\mathrm{Cl}: 1.14-6.65, P=0.024)$, and extraskeletal sites (HR=3.33, 95\% Cl: 1.02-10.85, $P=0.046)$.

Conclusions: This large population-based series described the clinical characteristics of malignant GCTB. Patients with stage T2/3 disease, distant disease and extra-skeletal sites had more odds to be with worse survival. The elder age than 34 years had a gradually increased risk for survival.

\section{Background}

Giant cell tumours of bone (GCTBs) are rare. It was reported that GCTBs accounted for approximately 5$6 \%$ of primary bone tumours [1]. The annual incidence was reported to be 1.7 (1.4-1.9) per million [2]. The metaphysis of long bones is the major affected site, and many studies have been conducted to investigate both the clinical characteristics [3, 4] and treatments [5].

Most GCTBs are thought to be pathologically benign cases. Some tumours showed aggressive features, such as cortical and subchondral involvement, reactive bony zone infiltration, and soft tissue masses [6, 7]. Recently, a study including four large series identified 92 cases of malignant GCTB in 2,315 patients; the incidence ranged from $1.1-11.3 \%$, and the cumulative incidence was $4.0 \%$ [8]. It is important to perform survival prediction to determine individual treatments. However, the issues have not been intensively studied due to the lack of clinical long-term follow-up data [9]. Itkin and his colleagues retrospectively retrieved the data of 242 patients from 26 studies with a median follow-up of 6.9 years [10]. They reported an estimated overall survival rate of $86.9 \%$. Few studies have investigated the prognostic factors of patients with malignant GCTB. 
The Surveillance, Epidemiology, and End Results (SEER) database is one of the largest cancer databases in the USA. Based on this database, a previous study estimated the survival among 117 malignant GCTB patients seen from 1975 to 2004 . The prognostic factors for poor survival included older age and distant metastasis at diagnosis [11].

This database has been extended, and some new factors have been added, including TNM stages, updated AJCC grade, insurance status, and records of specific metastatic organs. The updated data should be re-evaluated to provide further understanding of malignant GCTB. In the present study, based on the updated data from the database, we attempted to evaluate the survival of patients with malignant GCTB and to identify prognostic factors.

\section{Methods}

\section{Data Source}

Data from patients with malignant GCTB were extracted from the SEER database with SEER ${ }^{\star}$ Stat Software version 8.3.5 (https://seer.cancer.gov/seerstat/) (Information Management Service, Inc. Calverton, MD, USA). According to the ICD-0-3/WH02008 guidelines, data from patients who were diagnosed with malignant GCTB (code: 9250/3: giant cell tumour of bone, malignant) between 1975 and 2016 were collected. Patients were excluded if they were diagnosed at autopsy or via death certificate. Malignant GCTBs located in extraskeletal sites such as the kidney, lung, pancreas and thyroid were excluded.

\section{Statistical analysis}

From the records of patients in the SEER database, the following patient-related variables were selected for analysis. We investigated the relationships between age and diagnosis year as continuous measure and overall survival using non-linear restricted cubic splines (RCS) model with 3 knots. As reported by Harrell, the positions of knots were selected at $0.1,0.5$ and 0.9 quintiles of the distribution [12]. The age was divided into four sections ( $\leq 18,19-34,35-60$ or $>60$ years), and the year interval of diagnosis was divided into 1975-1984, 1985-2004, 2005-2013, and 2014-2016.

The other variables included sex (male or female); marital status (unmarried or married); race (white, black, Indian/Alaska Native (IA) or Asian or Pacific Islander (API)); primary site (long bones of lower limb with associated joints, long bones of upper limb with scapula and associated joints, pelvis and spine, irregular bone and other sites outside of bone); tumour grade (I, II, III, or IV); T stage (T1, T2, or T3); N stage (N0 or N1); M stage (M0 or M1); historic SEER stage (localized, regional, or distant); and surgical treatment of the primary site (none or yes). Patients without clear records were classified as unknown for the corresponding parameter.

Continuous data are described as the mean \pm standard deviation. Differences between groups were analysed with univariate analysis. Categorical data are presented as the number and percentage, and the 
difference was evaluated by Pearson's chi-square test. The 1-, 5-, and 10-year overall survival (OS) values were calculated by the Kaplan-Meier method, and the log-rank test was used to evaluate the differences. The variables that had a $P$ value $<0.05$ in the univariate Cox regression analysis and those that had a significant effect on the prognosis in the biologically based theoretical model were incorporated into the multivariate model. To evaluate the influence of extraskeletal sites on survival, a sensitivity analysis was performed by including or excluding cases.

All statistical analyses were performed using SPSS 23.0 (IBM Corporation, Armonk, NY, USA) and MedCalc 15.2.2. Two-sided $P$ value $<0.05$ was considered statistically significant.

\section{Results}

\section{Patient characteristics}

The selection process of the patients is illustrated in Fig. 1. According to the inclusion criteria, 334 patients with malignant GCTB seen from 1975 to 2016 were initially selected. After excluding one case that was diagnosed via death certificate and eight patients with extraskeletal GCTB, 325 patients were finally included. Table 1 shows the detailed clinicopathological features of the patients. The median age of the patients was 34 years, and a female predominance (52.3\%) was observed. More tumours were located in the long bones and associated joints (64.6\%) than in other locations, especially in the lower limbs (48.6\%). More than $50 \%$ of patients had no clear records of tumour grade, T stage, $\mathrm{N}$ stage or $\mathrm{M}$ stage. Twenty-two patients showed distant metastasis, including lung metastasis (10/22, 45.45\%), bone metastasis $(5 / 22,22.73 \%)$ and other metastasis $(7 / 22,31.82 \%)$. Regarding historic SEER stage, the localized and regional stages were the most frequent, occurring in $61.2 \%$ of all the patients, while $13.5 \%$ of patients had disease at distant stage. Surgery was performed in $53.85 \%$ of the patients. 
Table 1

Description of the patients with malignant giant cell tumor of bone.

\begin{tabular}{|c|c|c|}
\hline Subject characteristics & Number & Present (\%) \\
\hline \multicolumn{3}{|l|}{ Sex } \\
\hline Male & 155 & 47.69 \\
\hline Female & 170 & 52.31 \\
\hline \multicolumn{3}{|l|}{ Age } \\
\hline$\leq 18$ & 31 & 9.54 \\
\hline $19-34$ & 138 & 42.46 \\
\hline $35-60$ & 122 & 37.54 \\
\hline$>60$ & 34 & 10.46 \\
\hline \multicolumn{3}{|l|}{ Marital status } \\
\hline Unmarried & 162 & 49.85 \\
\hline Married & 136 & 41.85 \\
\hline Unknown & 27 & 8.31 \\
\hline \multicolumn{3}{|l|}{ Insurance recode } \\
\hline Uninsured & 113 & 34.77 \\
\hline Insured & 8 & 2.46 \\
\hline Unknown & 204 & 62.77 \\
\hline \multicolumn{3}{|l|}{ Race } \\
\hline White & 241 & 74.15 \\
\hline Black & 41 & 12.62 \\
\hline Al & 8 & 2.46 \\
\hline API & 28 & 8.62 \\
\hline Unknown & 7 & 2.15 \\
\hline \multicolumn{3}{|l|}{ Year of diagnosis } \\
\hline 1975-1984 & 31 & 9.54 \\
\hline $1985-2004$ & 132 & 40.62 \\
\hline $2005-2013$ & 126 & 38.77 \\
\hline
\end{tabular}

Note: IA: Indian/Alaska Native; API: Asian or Pacific Islander 


\begin{tabular}{|c|c|c|}
\hline Subject characteristics & Number & Present (\%) \\
\hline 2014-2016 & 36 & 11.08 \\
\hline \multicolumn{3}{|l|}{ Primary site } \\
\hline Long bones of lower limb and associated joints & 158 & 48.62 \\
\hline Long bones of upper limb and associated joints & 52 & 16.00 \\
\hline Pelvis and spine & 59 & 18.15 \\
\hline Irregular bone, short bone, and flat bone & 47 & 14.46 \\
\hline Unknown & 9 & 2.77 \\
\hline \multicolumn{3}{|l|}{ Grade } \\
\hline Grade I & 9 & 2.77 \\
\hline Grade II & 18 & 5.54 \\
\hline Grade III & 12 & 3.69 \\
\hline Grade IV & 31 & 9.54 \\
\hline Unknown & 255 & 78.46 \\
\hline \multicolumn{3}{|l|}{ T stage 2004+ } \\
\hline T1 & 61 & 18.77 \\
\hline T2 & 41 & 12.62 \\
\hline T3 & 4 & 1.23 \\
\hline Unknown & 219 & 67.38 \\
\hline \multicolumn{3}{|l|}{ N stage 2004+ } \\
\hline NO & 149 & 45.85 \\
\hline N1 & 1 & 0.31 \\
\hline Unknown & 175 & 53.85 \\
\hline \multicolumn{3}{|l|}{ M stage 2004+ } \\
\hline MO & 142 & 43.69 \\
\hline M1 & 22 & 6.77 \\
\hline Unknown & 161 & 49.54 \\
\hline
\end{tabular}

Note: IA: Indian/Alaska Native; API: Asian or Pacific Islander 


\begin{tabular}{|lll|}
\hline Subject characteristics & Number & Present (\%) \\
\hline Localized & 111 & 34.15 \\
\hline Regional & 88 & 27.08 \\
\hline Distant & 44 & 13.54 \\
\hline Unknown & 82 & 25.23 \\
\hline Surg (prim) 1998+ & & \\
\hline None & 59 & 18.15 \\
\hline Yes & 175 & 53.85 \\
\hline Unknown & 91 & 28.00 \\
\hline Note: IA: Indian/Alaska Native; API: Asian or Pacific Islander \\
\hline
\end{tabular}

Eight patients were diagnosed with extraskeletal GCTB. Their data were recorded from 1987 to 2016, and five patients were female. Two of the patients were diagnosed at approximately 20 years old, while the others were older than 50 years. Regarding the primary site, three cases were found in the pancreas, one was found in the kidney, one was found in the thyroid, one was found in the mediastinum, one was found in the lung, and there was one subcutaneous case. There was no clear record of tumour grade in four patients, one of whom had grade I disease, two of whom had grade III disease, and one of whom had grade IV disease. Two patients had long survival (135 and 136 months), one died at 24 months after diagnosis, and five patients died within 7 months.

\section{Survival analysis and prognostic factor identification}

The overall survival of the entire cohort is illustrated in Fig. 2. The overall 1-, 5-, and 10-year survival rates were $94.3 \%$ (95\% Cl: $91.7-96.8), 82.3 \%$ (95\% Cl: 77.9-86.6), and 80.1\% (95\% Cl: 75.4-84.7), respectively. The detailed OS data for patients with malignant GCTB according to each characteristic are summarized in Table 2. As shown in the Figs. $3 \mathrm{~A}$ and $3 \mathrm{~B}$, three knots were applied to the data and stratified the age and diagnosis year into four groups. We observed a potential non-linear J-shaped dose-response relationship between the age or diagnosis year and survival. Elder age had a gradually increased risk for survival. 
Table 2

The overall survival (OS) in patients with malignant giant cell tumor of bone diagnosed between 1975 and 2016.

\begin{tabular}{|c|c|c|c|c|}
\hline Factors & $\begin{array}{l}\text { 1-year OS, \% } \\
(95 \% \mathrm{Cl})\end{array}$ & $\begin{array}{l}\text { 5-year OS, \% } \\
\text { (95\% Cl) }\end{array}$ & $\begin{array}{l}\text { 10-year OS, \% } \\
(95 \% \mathrm{Cl})\end{array}$ & $\begin{array}{l}P \\
\text { value }\end{array}$ \\
\hline Sex & & & & 0.397 \\
\hline Male & $\begin{array}{l}95.4(92.0- \\
98.7)\end{array}$ & $\begin{array}{l}83.1(76.7- \\
89.3)\end{array}$ & $\begin{array}{l}79.2(72.1- \\
86.2)\end{array}$ & \\
\hline Female & $\begin{array}{l}93.3(89.5- \\
97.0)\end{array}$ & $\begin{array}{l}81.6(75.5- \\
87.5)\end{array}$ & $\begin{array}{l}80.8(74.6- \\
86.9)\end{array}$ & \\
\hline Age & & & & <.001 \\
\hline$\leq 18$ & $\begin{array}{l}98.8(90.5- \\
100.0)\end{array}$ & NA & NA & \\
\hline $19-34$ & $\begin{array}{l}99.3(97.9- \\
100.0)\end{array}$ & $\begin{array}{l}91.9(87.0- \\
96.8)\end{array}$ & NA & \\
\hline $35-60$ & $\begin{array}{l}91.4(86.3- \\
96.5)\end{array}$ & $\begin{array}{l}79.2(71.6- \\
86.8)\end{array}$ & $\begin{array}{l}75.7(67.5- \\
83.9)\end{array}$ & \\
\hline$>60$ & $\begin{array}{l}81.3(67.8- \\
94.8)\end{array}$ & $\begin{array}{l}33.9(15.1- \\
52.7)\end{array}$ & $22.6(4.6-40.6)$ & \\
\hline Marital status & & & & 0.307 \\
\hline Unmarried & $\begin{array}{l}95.6(92.4- \\
98.7)\end{array}$ & $\begin{array}{l}82.9(76.7- \\
88.9)\end{array}$ & NA & \\
\hline Married & $\begin{array}{l}92.2(87.6- \\
96.8)\end{array}$ & $\begin{array}{l}79.5(72.3- \\
86.5)\end{array}$ & $\begin{array}{l}75.5(67.7- \\
83.2)\end{array}$ & \\
\hline Unknown & $\begin{array}{l}96.2(88.8- \\
100.0)\end{array}$ & & $\begin{array}{l}87.0(73.1- \\
100.0)\end{array}$ & \\
\hline Insurance recode & & & & 0.251 \\
\hline Uninsured & $\begin{array}{l}92.5(87.5- \\
97.4)\end{array}$ & $\begin{array}{l}76.7(67.7- \\
85.5)\end{array}$ & $\begin{array}{l}74.0(63.9- \\
83.9)\end{array}$ & \\
\hline Insured & & NA & NA & \\
\hline Unknown & $\begin{array}{l}95.0(92.0- \\
97.9)\end{array}$ & $\begin{array}{l}84.7(79.7- \\
89.6)\end{array}$ & $\begin{array}{l}82.6(77.3- \\
87.8)\end{array}$ & \\
\hline Race & & & & 0.560 \\
\hline White & $\begin{array}{l}93.5(90.3- \\
96.6)\end{array}$ & $\begin{array}{l}82.5(77.4- \\
87.5)\end{array}$ & $\begin{array}{l}80.1(74.6- \\
85.5)\end{array}$ & \\
\hline Black & $\begin{array}{l}97.5(92.7- \\
100.0)\end{array}$ & $\begin{array}{l}81.9(69.7- \\
93.8)\end{array}$ & NA & \\
\hline
\end{tabular}




\begin{tabular}{|c|c|c|c|c|}
\hline Factors & $\begin{array}{l}\text { 1-year OS, \% } \\
(95 \% \mathrm{Cl})\end{array}$ & $\begin{array}{l}\text { 5-year OS, \% } \\
\text { (95\% Cl) }\end{array}$ & $\begin{array}{l}\text { 10-year OS, \% } \\
(95 \% \mathrm{Cl})\end{array}$ & $\begin{array}{l}P \\
\text { value }\end{array}$ \\
\hline $\mathrm{Al}$ & & $\begin{array}{l}75.0(45.0- \\
100.0)\end{array}$ & NA & \\
\hline API & $\begin{array}{l}89.0(77.2- \\
100.0)\end{array}$ & $\begin{array}{l}80.3(64.7- \\
95.6)\end{array}$ & $\begin{array}{l}73.0(53.4- \\
92.2)\end{array}$ & \\
\hline \multicolumn{5}{|l|}{ Unknown } \\
\hline Year of diagnosis & & & & 0.156 \\
\hline $1975-1984$ & $\begin{array}{l}90.3(79.9- \\
100.0)\end{array}$ & $\begin{array}{l}87.1(75.3- \\
98.9)\end{array}$ & NA & \\
\hline $1985-2004$ & $\begin{array}{l}96.1(92.8- \\
99.4)\end{array}$ & $\begin{array}{l}87.4(81.5- \\
93.3)\end{array}$ & $\begin{array}{l}84.1(77.6- \\
90.6)\end{array}$ & \\
\hline $2005-2013$ & $\begin{array}{l}93.7(89.4- \\
98.0)\end{array}$ & $\begin{array}{l}75.9(68.3- \\
83.5)\end{array}$ & $\begin{array}{l}74.5(66.5- \\
82.5)\end{array}$ & \\
\hline $2014-2016$ & $\begin{array}{l}94.3(86.7- \\
100.0)\end{array}$ & $\begin{array}{l}88.0(74.1- \\
100.0)\end{array}$ & NA & \\
\hline Primary site & & & & 0.333 \\
\hline $\begin{array}{l}\text { Long bones of lower limb and } \\
\text { associated joints }\end{array}$ & $\begin{array}{l}94.7(91.1- \\
98.2)\end{array}$ & $\begin{array}{l}84.0(78.0- \\
89.9)\end{array}$ & $\begin{array}{l}81.3(74.7- \\
87.7)\end{array}$ & \\
\hline $\begin{array}{l}\text { Long bones of upper limb and } \\
\text { associated joints }\end{array}$ & $\begin{array}{l}98.0(94.0- \\
100.0)\end{array}$ & $\begin{array}{l}86.9(77.1- \\
96.5)\end{array}$ & $\begin{array}{l}84.3(73.5- \\
94.8)\end{array}$ & \\
\hline Pelvis and spine & $\begin{array}{l}94.8(89.1- \\
100.0)\end{array}$ & $\begin{array}{l}74.9(62.9- \\
86.6)\end{array}$ & NA & \\
\hline Irregular, short, and flat bone & $\begin{array}{l}91.2(82.9- \\
99.3)\end{array}$ & $\begin{array}{l}83.9(72.9- \\
94.7)\end{array}$ & $81.0(69.0-92.7)$ & \\
\hline Unknown & $\begin{array}{l}77.8(50.6- \\
100.0)\end{array}$ & $\begin{array}{l}64.8(32.5- \\
96.5)\end{array}$ & NA & \\
\hline Grade & & & & $\dot{0} 001$ \\
\hline Grade I & & NA & NA & \\
\hline Grade II & $\begin{array}{l}94.4(83.8- \\
100.0)\end{array}$ & $\begin{array}{l}81.9(63.2- \\
100.0)\end{array}$ & NA & \\
\hline Grade III & $\begin{array}{l}91.7(76.1- \\
100.0)\end{array}$ & $\begin{array}{l}60.0(27.3- \\
92.1)\end{array}$ & $40.0(1.2-78.0)$ & \\
\hline Grade IV & $\begin{array}{l}71.8(55.0- \\
88.3)\end{array}$ & $\begin{array}{l}53.8(34.0- \\
73.2)\end{array}$ & $\begin{array}{l}47.1(25.9- \\
67.8)\end{array}$ & \\
\hline Unknown & $\begin{array}{l}96.7(94.5- \\
98.9)\end{array}$ & $\begin{array}{l}86.1(81.6- \\
90.5)\end{array}$ & $\begin{array}{l}84.4(79.6- \\
89.1)\end{array}$ & \\
\hline
\end{tabular}




\begin{tabular}{|c|c|c|c|c|}
\hline Factors & $\begin{array}{l}\text { 1-year OS, \% } \\
\text { (95\% Cl) }\end{array}$ & $\begin{array}{l}\text { 5-year OS, \% } \\
\text { (95\% Cl) }\end{array}$ & $\begin{array}{l}\text { 10-year OS, \% } \\
(95 \% \mathrm{Cl})\end{array}$ & $\begin{array}{l}P \\
\text { value }\end{array}$ \\
\hline T stage 2004+ & & & & $<.001$ \\
\hline T1 & $\begin{array}{l}96.7(92.2- \\
100.0)\end{array}$ & $\begin{array}{l}92.8(86.0- \\
99.5)\end{array}$ & NA & \\
\hline T2 & $\begin{array}{l}84.4(72.9- \\
95.7)\end{array}$ & $\begin{array}{l}47.0(26.2- \\
67.4)\end{array}$ & NA & \\
\hline T3 & & $\begin{array}{l}25.0(-17.5- \\
66.7)\end{array}$ & NA & \\
\hline Unknown & $\begin{array}{l}95.3(92.5- \\
98.1)\end{array}$ & $\begin{array}{l}85.5(80.7- \\
90.2)\end{array}$ & $\begin{array}{l}82.8(77.6- \\
87.9)\end{array}$ & \\
\hline N stage 2004+ & & & & 0.050 \\
\hline NO & $\begin{array}{l}93.7(89.7- \\
97.6)\end{array}$ & $\begin{array}{l}76.2(68.6- \\
83.6)\end{array}$ & $\begin{array}{l}74.8(66.8- \\
82.6)\end{array}$ & \\
\hline N1 & NA & NA & NA & \\
\hline Unknown & $\begin{array}{l}94.7(91.3- \\
98.0)\end{array}$ & $\begin{array}{l}86.8(81.6- \\
91.8)\end{array}$ & $\begin{array}{l}84.2(78.6- \\
89.7)\end{array}$ & \\
\hline M stage 2004+ & & & & ¿́.001 \\
\hline MO & $\begin{array}{l}97.2(94.5- \\
99.9)\end{array}$ & $\begin{array}{l}81.3(74.1- \\
88.3)\end{array}$ & $\begin{array}{l}79.8(72.2- \\
87.3)\end{array}$ & \\
\hline M1 & $\begin{array}{l}75.4(56.6- \\
93.8)\end{array}$ & $\begin{array}{l}53.9(31.6- \\
75.8)\end{array}$ & NA & \\
\hline Unknown & $\begin{array}{l}94.3(90.7- \\
97.9)\end{array}$ & $\begin{array}{l}86.5(81.1- \\
91.8)\end{array}$ & $83.8(78.0-89.5)$ & \\
\hline SEER historic stage & & & & <.001 \\
\hline Localized & $\begin{array}{l}97.3(94.3- \\
100.0)\end{array}$ & $\begin{array}{l}87.4(81.0- \\
93.7)\end{array}$ & $\begin{array}{l}86.1(79.2- \\
92.8)\end{array}$ & \\
\hline Regional & $\begin{array}{l}94.2(89.3- \\
99.0)\end{array}$ & $\begin{array}{l}80.0(71.1- \\
88.7)\end{array}$ & $\begin{array}{l}78.1(68.7- \\
87.3)\end{array}$ & \\
\hline Distant & $\begin{array}{l}84.0(73.1- \\
94.6)\end{array}$ & $\begin{array}{l}65.2(50.9- \\
79.2)\end{array}$ & $\begin{array}{l}59.4(44.3- \\
74.2)\end{array}$ & \\
\hline Unknown & $\begin{array}{l}96.0(91.5- \\
100.0)\end{array}$ & $\begin{array}{l}87.9(80.0- \\
95.6)\end{array}$ & $\begin{array}{l}86.2(77.8- \\
94.5)\end{array}$ & \\
\hline Surg (prim) 1998+ & & & & 0.024 \\
\hline
\end{tabular}




\begin{tabular}{|c|c|c|c|c|}
\hline Factors & $\begin{array}{l}\text { 1-year OS, \% } \\
(95 \% \mathrm{Cl})\end{array}$ & $\begin{array}{l}\text { 5-year OS, \% } \\
(95 \% \mathrm{Cl})\end{array}$ & $\begin{array}{l}\text { 10-year OS, \% } \\
(95 \% \text { Cl) }\end{array}$ & $\begin{array}{l}P \\
\text { value }\end{array}$ \\
\hline None & $\begin{array}{l}90.9(83.3- \\
98.3)\end{array}$ & $\begin{array}{l}70.3(56.9- \\
83.4)\end{array}$ & $\begin{array}{l}67.0(52.7- \\
81.0)\end{array}$ & \\
\hline Yes & $\begin{array}{l}94.7(91.3- \\
98.0)\end{array}$ & $\begin{array}{l}82.3(76.3- \\
88.2)\end{array}$ & $\begin{array}{l}80.2(73.7- \\
86.6)\end{array}$ & \\
\hline Unknown & $\begin{array}{l}95.5(91.2- \\
99.7)\end{array}$ & $\begin{array}{l}88.7(82.1- \\
95.2)\end{array}$ & $\begin{array}{l}86.4(79.2- \\
93.4)\end{array}$ & \\
\hline
\end{tabular}

The results of the univariate and multivariate Cox regression analyses are summarized in Table 3 . A lower OS was noted in patients with age from 35 to 60 years $(P=0.025)$ and patients older than 60 years $(P<$ $0.001)$, with grade IV disease $(P=0.041)$, with stage T2/3 disease $(P<0.001)$, with unknown $\mathrm{N}$ stage $(P=$ $0.019)$, with stage $\mathrm{M} 1$ disease $(P<0.001)$, with a distant historic SEER stage $(P<0.001)$, and who had not undergone surgery for the primary tumour $(P=0.05)$ under univariate analysis. Multivariate Cox regression analysis showed poor survival in patients with age from 35 to 60 years (hazard ratio $(H R)=$ 9.99, 95\% Cl: $1.34-74.80, P=0.025)$, age older than 60 years $(\mathrm{HR}=62.03,95 \% \mathrm{Cl}: 7.94-484.38, P<0.001)$, with stage $\mathrm{T} 2$ disease $(\mathrm{HR}=4.85,95 \% \mathrm{Cl}: 1.52-15.47, P=0.008)$, with stage T3 disease $(\mathrm{HR}=6.09,95 \%$ $\mathrm{Cl}: 1.03-36.23, P=0.047)$, and with distant tumours (HR=2.76,95\% $\mathrm{Cl}: 1.14-6.65, P=0.024)$. The sensitivity analysis suggested that a primary location of GCTB in extraskeletal sites was associated with poor survival ( $\mathrm{HR}=3.33,95 \% \mathrm{Cl}: 1.02-10.85, P=0.046)$. Analysis including the extraskeletal cases did not produce significantly different results (supplementary table). 
Table 3

Univariate and multivariate Cox regression analyzing the prognostic factors for survival of patients with malignant giant cell tumor of bone (1975 and 2016).

\begin{tabular}{|c|c|c|c|c|}
\hline \multirow[t]{2}{*}{ Subject characteristics } & \multicolumn{2}{|l|}{ Univariate } & \multicolumn{2}{|l|}{ Multivariate } \\
\hline & $\mathrm{HR}(95 \% \mathrm{Cl})$ & $\begin{array}{l}\mathrm{P} \text { - } \\
\text { value }\end{array}$ & $\mathrm{HR}(95 \% \mathrm{Cl})$ & $\begin{array}{l}\mathrm{P} \text { - } \\
\text { value }\end{array}$ \\
\hline \multicolumn{5}{|l|}{ Sex } \\
\hline Male & 1.00(Reference) & & 1.00(Reference) & \\
\hline Female & $0.82(0.51-1.31)$ & 0.399 & $0.79(0.45-1.38)$ & 0.400 \\
\hline \multicolumn{5}{|l|}{ Age } \\
\hline$\leq 18$ & 1.00(Reference) & & 1.00(Reference) & \\
\hline $19-34$ & $\begin{array}{l}3.72(0.49- \\
28.17)\end{array}$ & 0.204 & $\begin{array}{l}3.92(0.51- \\
30.18)\end{array}$ & 0.190 \\
\hline $35-60$ & $\begin{array}{l}11.23(1.53- \\
82.43)\end{array}$ & 0.017 & $\begin{array}{l}9.99(1.34- \\
74.80)\end{array}$ & 0.025 \\
\hline$>60$ & $\begin{array}{l}54.08(7.20- \\
406.21)\end{array}$ & $\begin{array}{l}<.001 \\
0.001\end{array}$ & $\begin{array}{l}62.03(7.94- \\
484.38)\end{array}$ & $\dot{0} 001$ \\
\hline \multicolumn{5}{|l|}{ Marital status } \\
\hline Unmarried & 1.00(Reference) & & NA & $N A$ \\
\hline Married & $1.47(0.90-2.42)$ & 0.127 & NA & $N A$ \\
\hline Unknown & $1.23(0.54-2.82)$ & 0.623 & NA & $N A$ \\
\hline \multicolumn{5}{|l|}{ Insurance recode } \\
\hline Uninsured & 1.00(Reference) & & & \\
\hline Insured & $0.74(0.10-5.47)$ & 0.765 & NA & $N A$ \\
\hline Unknown & $0.63(0.37-1.09)$ & 0.100 & NA & $N A$ \\
\hline \multicolumn{5}{|l|}{ Race } \\
\hline White & 1.00(Reference) & & & \\
\hline Black & $0.96(0.47-1.94)$ & 0.902 & NA & $N A$ \\
\hline $\mathrm{Al}$ & $1.07(0.26-4.42)$ & 0.920 & NA & $N A$ \\
\hline API & $1.54(0.73-3.24)$ & 0.258 & NA & $N A$ \\
\hline
\end{tabular}

Abbreviations: Met $=$ Metastases . 
Subject characteristics
Univariate

$\mathrm{HR}(95 \% \mathrm{Cl})$
Pvalue
Multivariate

$\mathrm{HR}(95 \% \mathrm{Cl})$
Pvalue

\section{Year of diagnosis}

1975-1984

1985-2004

2005-2013

2014-2016

\section{Primary site}

Long bones of lower limb and associated joints

Long bones of upper limb and associated joints

Pelvis and spine

Irregular bone, short bone, and flat bone

Unknown

\section{Grade}

Grade I

Grade II

Grade III

Grade IV

Unknown

\section{T stage}

T1

T2

T3
1.00(Reference)

$1.31(0.58-2.97) \quad 0.521$

$2.26(0.94-5.42) \quad 0.069$

1.98(0.49-8.05)
1.00(Reference)

$0.78(0.30-2.07) \quad 0.620$

$0.90(0.20-4.13) \quad 0.894$

$0.342 \quad 0.33(0.04-2.69) \quad 0.302$

1.00(Reference)

1.00(Reference)

$0.67(0.31-1.46)$

0.317

$0.63(0.27-1.46)$

0.278

$1.42(0.77-2.62)$

0.263

$1.03(0.52-2.06)$

0.933

$1.25(0.63-2.47)$

0.529

1.16(0.54-2.50)

0.697

$2.02(0.62-6.59)$

0.245

1.16(0.26-5.14)

0.842

1.00(Reference)

1.00(Reference)

$2.14(0.25-$

18.45)

0.488

2.10(0.22-

20.34)

$5.47(0.64-$

47.04)

0.121

3.87(0.41-

36.73)

8.37 (1.09-

64.19)

0.041

4.24(0.49-

36.95)

$1.70(0.23-$

12.38)

0.599

1.96(0.25-

15.34)
1.00(Reference)

$8.10(2.68-$

24.48)

16.07 (3.55-

72.68)
1.00(Reference)

$5.87(1.74-$

0.004

¿.001 19.86)

$<\quad 6.09(1.03-$

$0.001 \quad 36.23)$

0.047

Abbreviations: Met $=$ Metastases . 


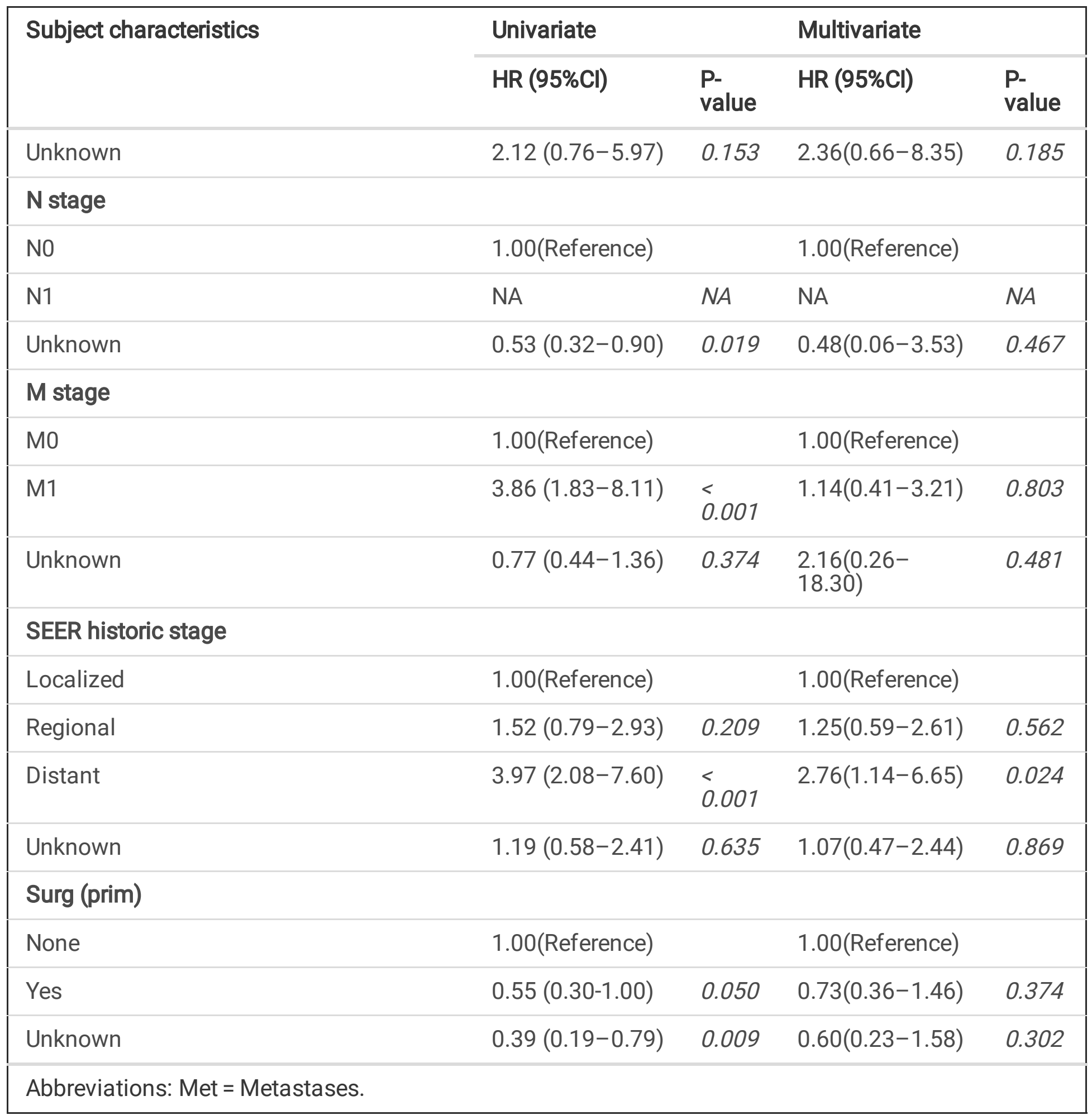

\section{Discussion}

In the present study, with the large cohort of patients with malignant GCTB, the 5-year OS was $82.3 \%$ (95\% Cl: $77.9-86.6)$. This rate was consistent with the rate of $86.9 \%$ reported by Itkin et al. [10] and $80 \%$ reported by Domovitov et al. [13]. Prognostic factors were identified, and poor survival was correlated with the following factors: age older than 34 years, high $\mathrm{T}$ stage, distant tumour stage and extraskeletal sites. 
Similar to the survival trend from the previous SEER cohort analysis [11], in the present study, poor survival was found in older patients and those with a historic SEER stage of distant. Data regarding metastatic sites have been added to the SEER dataset since 2010. Compared with the 5-year OS of $81.3 \%$ for patients without metastasis, that of patients with metastasis significantly decreased to $53.9 \%$. Previous studies reported that the incidence of lung metastasis in benign giant cell tumours was approximately $3.5 \%[14,15]$, while it was approximately $8 \%$ in malignant cases $[16,17]$. In our study, 14 out of 89 cases extracted from 2010 to 2016 were recorded as M1 status, and the lung was the major metastatic site (10 out of 89 cases, $11.2 \%$ ). The higher incidence of lung metastasis in this study than in the previous study may be a result of the introduction of regular screening. More lung metastases were reported to develop early after the initial diagnosis of GCTB and after recurrence than long after diagnosis $[17,18]$. Screening for possible metastasis should especially be performed in these situations.

TNM classification data have been added to the SEER database since 2004, and the results showed a trend for poor survival in patients with higher $\mathrm{T}$ stage or higher $\mathrm{N}$ stage. The multivariate Cox regression analysis suggested that higher $\mathrm{T}$ stage was a prognostic factor. Tumour size was previously found to be an independent risk factor for lung metastasis [19]. Based on the Campanacci classification, which has been widely accepted for evaluating giant cell tumours, grade III tumours are the aggressive type. A series of studies reported Campanacci grade III as a risk factor for tumour recurrence [20,21] and lung metastasis [22, 23]. Recurrence was also reported to be a risk factor for metastasis $[22,23]$. Therefore, $T$ stage and/or Campanacci classification played an important role in evaluating the survival of patients with giant cell tumours.

To our knowledge, this is the first study of extraskeletal malignant GCTB. Based on our results, an extraskeletal site was associated with poor survival. Only two patients lived longer than 11 years, while five patients died in less than seven months. Therefore, attention should be paid to patients with lesions in extraskeletal sites. Our study was limited by the few available cases, and further analysis revealing potential prognostic factors in a large population could not be conducted. More cases from multiple centres will be needed.

For treating giant cell tumours, both resection with reconstruction and intralesional curettage have been widely accepted [24-26]. Surgery with/without radiotherapy did not significantly improve survival in a previous SEER study [11]. Similarly, surgery was not an independent survival factor of the patients with malignant GCTB in the present study. The effect of surgery on the survival of patients with malignant GCTB was different from its effect in other malignant cancers, such as colorectal cancer [27] and ovarian cancer[28]. This difference may be caused by the significantly higher survival in giant cell tumours than in other malignant cancers.

Undoubtedly, there are some limitations in the present study. More than $50 \%$ of patients had no clear records of tumour grade, $\mathrm{T}$ stage, $\mathrm{N}$ stage or $\mathrm{M}$ stage. More clear data are needed to further reveal the effect of AJCC grade and TNM stage on survival. As our study was limited by the absent records regarding surgery in the SEER database, further analysis of the correlation between surgery and 
recurrence or metastasis could not be performed. The results of the present study need further external validation.

\section{Conclusions}

In conclusion, based on the large population from the SEER database, the clinical characteristics and prognostic factors among patients with malignant GCTB were analysed. Stage T2/3 disease, distant stage and extraskeletal sites were possibly associated with worse survival in patients with malignant GCTB. There was an increased risk of elder age for survival after 34 years old.

\section{Abbreviations}

GCTB

giant cell tumour of bone

SEER

Surveillance, Epidemiology, and End Results database

AJCC

American Joint Committee on Cancer

OS

overall survival

HR

hazard ratio

$\mathrm{Cl}$

confidence interval

\section{Declarations}

\section{Ethics approval and consent to participate}

The data released from the public SEER database do not require patients' consent because cancer is a reportable disease in every state of the United States. The present study complied with the 1964 Helsinki Declaration and its later amendments or comparable ethical standards.

\section{Consent for publication}

Not applicable

\section{Availability of data and material}

Data from patients with malignant GCTB were extracted from the SEER database with SEER ${ }^{\star}$ Stat Software version 8.3.5 (https://seer.cancer.gov/seerstat/) (Information Management Service, Inc. Calverton, MD, USA). 


\section{Competing interests}

All the authors report no conflicts of interest in this work.

\section{Funding}

The present study was sponsored by Natural Science Foundation of China $(81702161,8191101553$, $81802508,81903398)$, Top talent training program of the first affiliated hospital of PLA Army Medical University (SWH2018BJKJ-12), Laboratory of Tumor Immunology and Pathology (Army Medical University), Ministry of Education (2017jszl01).

\section{Authors' contributions}

JZ, MM and CZ made contributions to the conception of this study. GX, HW and JD made contributed to acquisition of data and interpretation of results. XW and FL performed statistical analysis. FL, HW and JD prepared the tables and figures. JZ and MM mainly prepared the manuscript. GX and CZ reviewed and reedited the manuscript. All authors read and approved the final manuscript.

\section{Acknowledgements}

Not Applicable

\section{References}

[1] Lopez-Pousa A, Martin BJ, Garrido T, Vazquez J. Giant cell tumour of bone: new treatments in development. Clin TransI Oncol. 2015;17:419-30.

[2] Verschoor AJ, Bovee J, Mastboom M, Sander DP, Van De Sande M, Gelderblom H. Incidence and demographics of giant cell tumor of bone in The Netherlands: First nationwide Pathology Registry Study. Acta Orthop. 2018;89:570-4.

[3] Errani C, Ruggieri P, Asenzio MA, Toscano A, Colangeli S, Rimondi E, et al. Giant cell tumor of the extremity: A review of 349 cases from a single institution. Cancer Treat Rev. 2010;36:1-7.

[4] Zou C, Lin T, Wang B, Zhao Z, Li B, Xie X, et al. Managements of giant cell tumor within the distal radius: A retrospective study of 58 cases from a single center. J Bone Oncol. 2019;14:100211.

[5] van der Heijden L, Dijkstra PD, Campanacci DA, Gibbons CL, van de Sande MA. Giant cell tumor with pathologic fracture: should we curette or resect? Clin Orthop Relat Res. 2013;471:820-9.

[6] Present D, Bertoni F, Hudson T, Enneking WF. The correlation between the radiologic staging studies and histopathologic findings in aggressive stage 3 giant cell tumor of bone. Cancer-Am Cancer Soc. 1986;57:237-44.

[7] Chakarun CJ, Forrester DM, Gottsegen CJ, Patel DB, White EA, Matcuk GJ. Giant cell tumor of bone: review, mimics, and new developments in treatment. Radiographics. 2013;33:197-211. 
[8] Palmerini E, Picci P, Reichardt P, Downey G. Malignancy in Giant Cell Tumor of Bone: A Review of the Literature. Technol Cancer Res Treat. 2019;18:1078107648.

[9] Gong L, Liu W, Sun X, Sajdik C, Tian X, Niu X, et al. Histological and clinical characteristics of malignant giant cell tumor of bone. Virchows Arch. 2012;460:327-34.

[10] Itkin B, Straminsky S, De Ronato G, Lewi D, Marantz A, Bardach A. Prognosis of metastatic giant cell tumor of bone in the pre-denosumab era. A systematic review and a meta-analysis. Jpn J Clin Oncol. 2018;48:640-52.

[11] Beebe-Dimmer JL, Cetin K, Fryzek JP, Schuetze SM, Schwartz K. The epidemiology of malignant giant cell tumors of bone: an analysis of data from the Surveillance, Epidemiology and End Results Program (1975-2004). Rare Tumors. 2009;1:e52.

[12] Jr FEH. Regression Modeling Strategies: With Applications to Linear Models, Logistic Regression, and Survival Analysis. 2010:2-26.

[13] Domovitov SV, Healey JH. Primary malignant giant-cell tumor of bone has high survival rate. Ann Surg Oncol. 2010;17:694-701.

[14] Junior RC, Pereira MG, Garcia PB, Santos PA, Cavalcanti AS, Meohas W. Epidemiological study on giant cell tumor recurrence at the Brazilian National Institute of Traumatology and Orthopedics. Rev Bras Ortop. 2016;51:459-65.

[15] Niu X, Zhang Q, Hao L, Ding Y, Li Y, Xu H, et al. Giant cell tumor of the extremity: retrospective analysis of 621 Chinese patients from one institution. J Bone Joint Surg Am. 2012;94:461-7.

[16] Jiang N, Qin $\mathrm{CH}$, Tan CX, Wen SF, Ma YF, Dong F, et al. A retrospective analysis of 140 patients with giant cell tumor in the extremity: a multicenter study based on four hospitals in South China. Cancer Epidemiol. 2013;37:294-9.

[17] Rosario M, Kim HS, Yun JY, Han I. Surveillance for lung metastasis from giant cell tumor of bone. J Surg Oncol. 2017;116:907-13.

[18] Dominkus M, Ruggieri P, Bertoni F, Briccoli A, Picci P, Rocca M, et al. Histologically verified lung metastases in benign giant cell tumours-14 cases from a single institution. Int Orthop. 2006;30:499-504.

[19] Wang B, Chen W, Xie X, Tu J, Huang G, Zou C, et al. Development and validation of a prognostic index to predict pulmonary metastasis of giant cell tumor of bone. Oncotarget. 2017;8:108054-63.

[20] Urakawa H, Yonemoto T, Matsumoto S, Takagi T, Asanuma K, Watanuki M, et al. Clinical outcome of primary giant cell tumor of bone after curettage with or without perioperative denosumab in Japan: from a questionnaire for JCOG 1610 study. World J Surg Oncol. 2018;16:160. 
[21] Cheng DD, Hu T, Zhang HZ, Huang J, Yang QC. Factors Affecting the Recurrence of Giant Cell Tumor of Bone After Surgery: A Clinicopathological Study of 80 Cases from a Single Center. Cell Physiol Biochem. 2015;36:1961-70.

[22] Yang Y, Huang Z, Niu X, Xu H, Li Y, Liu W. Clinical characteristics and risk factors analysis of lung metastasis of benign giant cell tumor of bone. J Bone Oncol. 2017;7:23-8.

[23] Kito M, Matusmoto S, Ae K, Tanizawa T, Gokita T, Kobayashi H, et al. Pulmonary metastasis from giant cell tumor of bone: clinical outcome prior to the introduction of molecular target therapy. Jpn J Clin Oncol. 2017;47:529-34.

[24] Pazionis TJ, Alradwan H, Deheshi BM, Turcotte R, Farrokhyar F, Ghert M. A Systematic Review and Meta-Analysis of En-Bloc vs Intralesional Resection for Giant Cell Tumor of Bone of the Distal Radius. Open Orthop J. 2013;7:103-8.

[25] Zhang S, Zhang J, Wang X. Comparison of tumor curettage and resection for treatment of giant cell tumor of the bone around the knee joint. Pak J Med Sci. 2016;32:662-6.

[26] Charest-Morin R, Fisher CG, Varga PP, Gokaslan ZL, Rhines LD, Reynolds JJ, et al. En Bloc Resection Versus Intralesional Surgery in the Treatment of Giant Cell Tumor of the Spine. Spine (Phila Pa 1976). 2017;42:1383-90.

[27] Guo X, Zhang C, Ma W, Tian F, Xu G, Han X, et al. Patterns of bone metastases in newly diagnosed colorectal cancer: a real-world analysis in the SEER database. Int J Colorectal Dis. 2019.

[28] Zhang C, Guo X, Peltzer K, Ma W, Qi L, Zhang Y, et al. The prevalence, associated factors for bone metastases development and prognosis in newly diagnosed ovarian cancer: a large population based real-world study. J Cancer. 2019;10:3133-9.

\section{Figures}


Malignant giant cell tumor of bone diagnosed from 1975 to 2016

$(\mathbf{N}=\mathbf{3 3 4})$

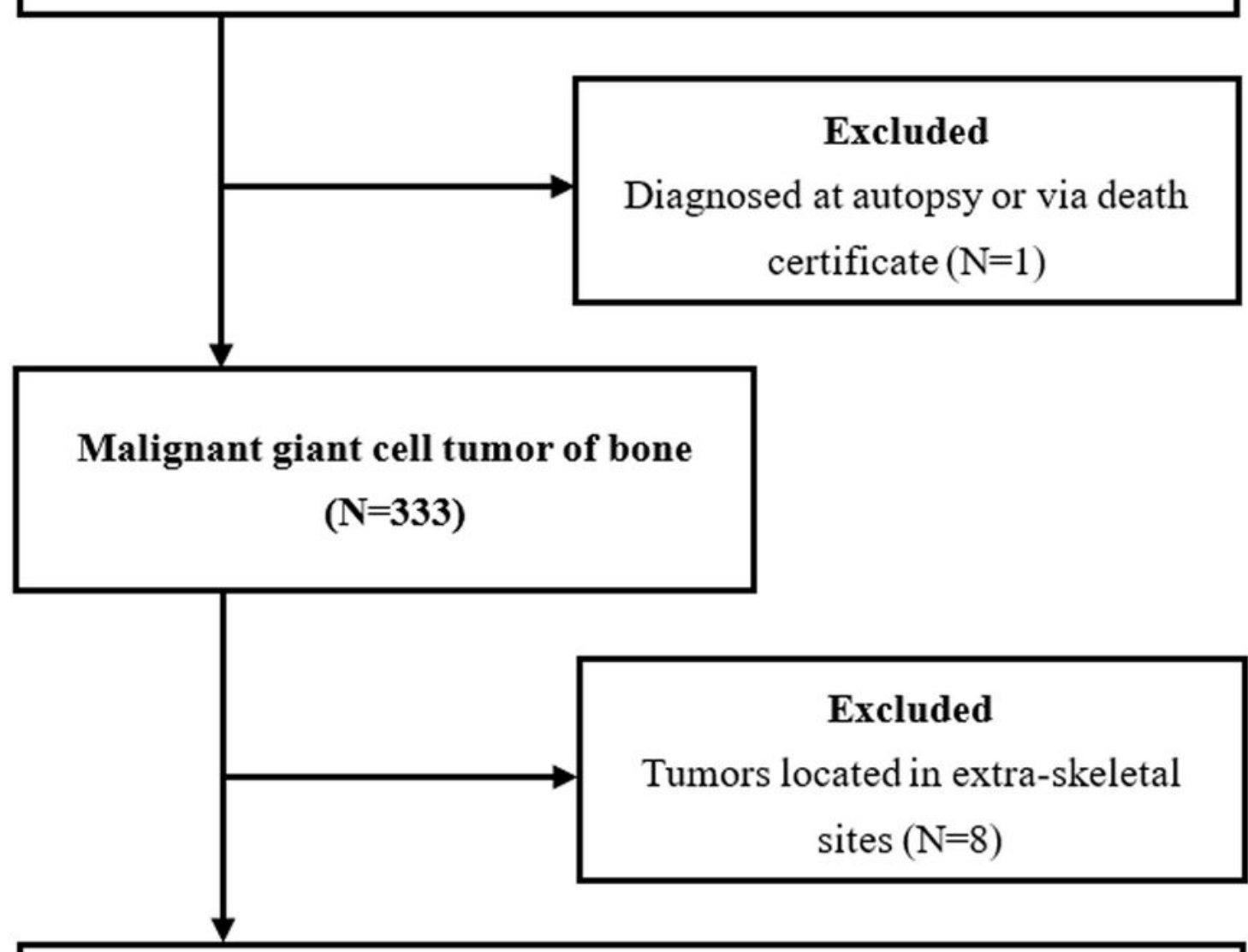

Giant cell tumor of bone originated from bones and joints:

Positive histology $(\mathrm{N}=316)$

Radiography without microscopic confirm $(\mathrm{N}=5)$

Positive exfoliative cytology, no positive histology $(\mathrm{N}=2)$

Positive microscopic confirm, method not specified $(\mathrm{N}=1)$

Unknown $(\mathrm{N}=1)$

All of these patients were included in the univariable and multivariable regression analysis $(\mathrm{N}=325)$

Figure 1

Process of patient selection for analysing both the survival and prognostic factors of patients with malignant GCTB. 


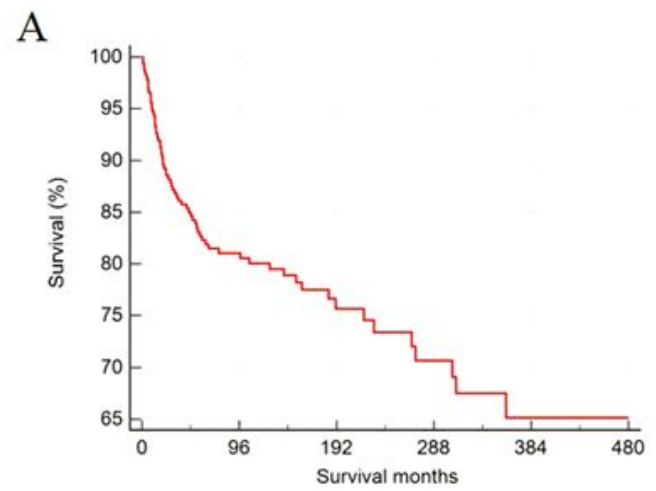

$\mathrm{B}$

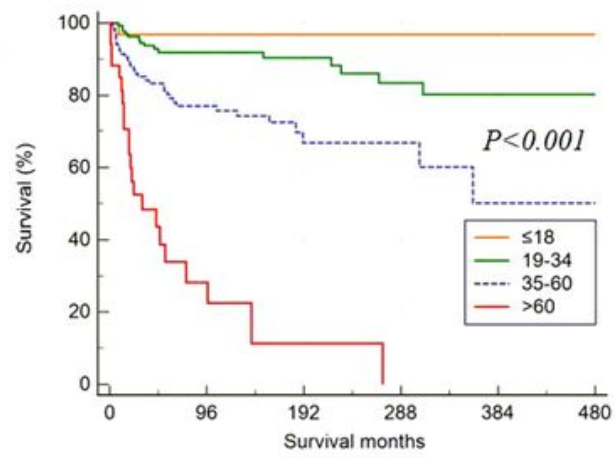

D

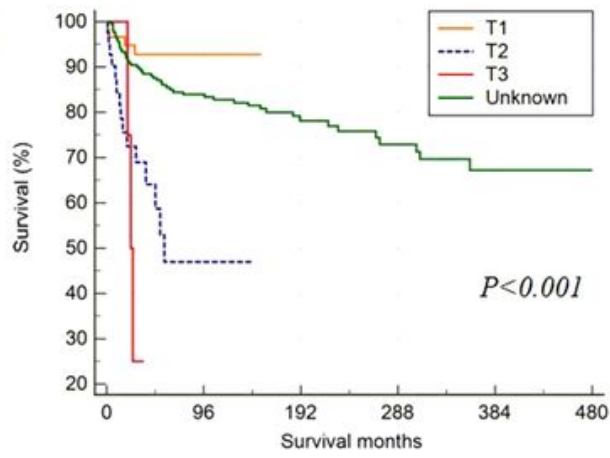

$\mathrm{F}$

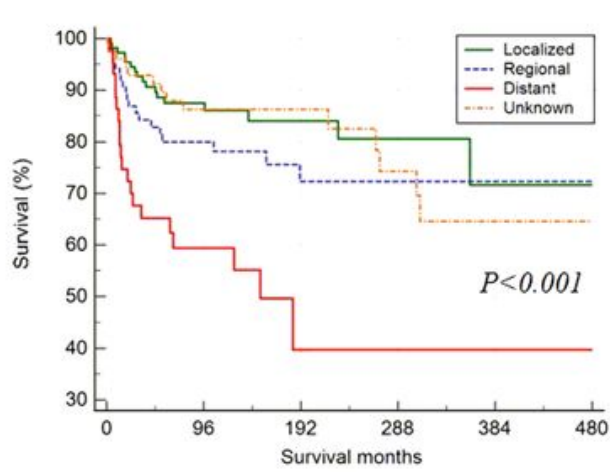

$\mathrm{C}$

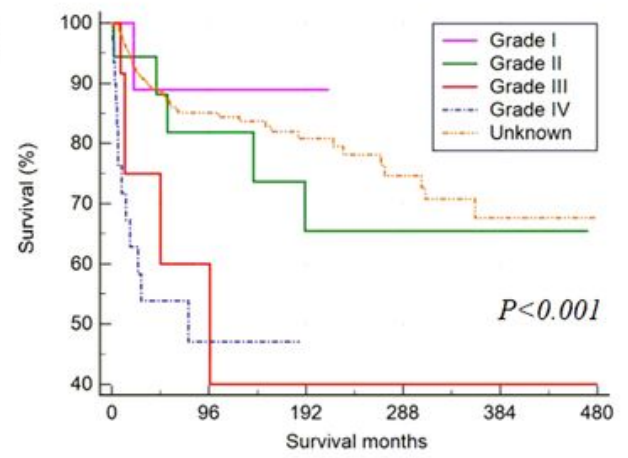

E

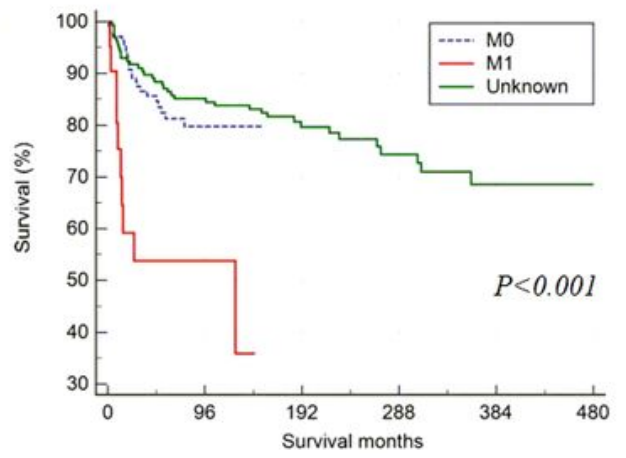

G

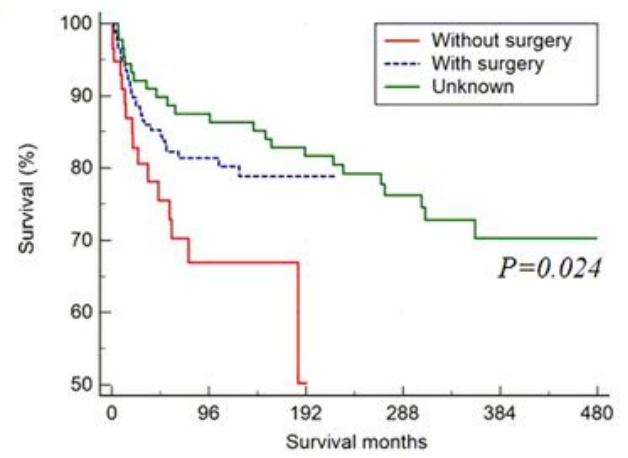

Figure 2

The overall survival of patients with GCTB from the SEER database. 


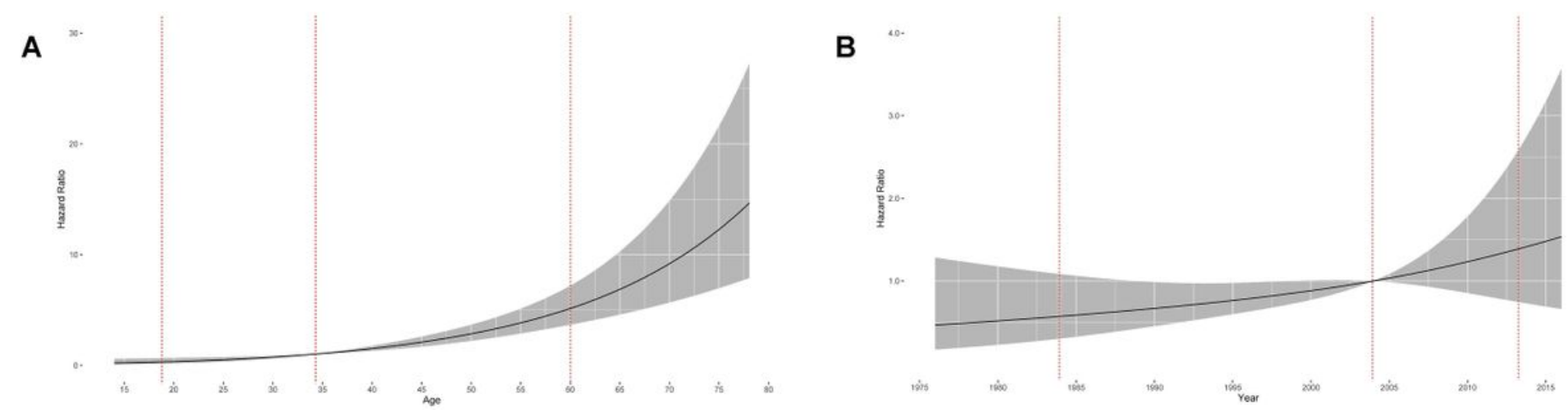

\section{Figure 3}

The trend of survival of patients with malignant GCTB on age (A) and diagnosis year (B).

\section{Supplementary Files}

This is a list of supplementary files associated with this preprint. Click to download.

- SupplementaryTable.docx

- SupplementaryTable.docx 